\title{
Neuroendocrinology 1965-66;1:369-371
}

\section{Subject Index}

ACTH;

-, EEG, effect on 144

-, sexual behavior, effect on 144

Amphibians, metamorphosis, hormonal

regulation of 45, 293 Anesthetic agents, pituitary-adrenocorti-

cal system, effect on 184 Antidiuretic hormone, blood flow, effect

on 23 Autonomic nervous system, calcification,

induction of 240 Avoidance conditioning; sexual

behavior 144

Blood flow, vasopressinn, effect on 23 Books; list of books received 192, 312 -; reviews 364 Brain, 5-hydroxytryptamine in 251

Calcification of autonomic nervous

system, induction or 240 Corticoid-feedback 228 Corticoids; implantation in

hypothalamus 129, 228 Corticosterone secretion, thyroxine,

effect on 15 Corticotrophin-releasing factor,

separation of 341 Cortisol; -, 5 -hydroxytryptamine in brain and

pineal, effect on 251 -, liver enzymes, effect on 138 -, sexual behavior, effect on 144

Depressive illness, dexamethasone, effect

on 358 Dexamethasone, effect of, in depressive

on 358 Dexame
illness 358

EEG;

, effect of ACTH on 144

rat 31 Electrical shock, effects of, in man 105 Endocraniosis 68 Enzymes (transaminases) in liver,

effect of stress and cortisol on 138 Epinephrine excretion in man, effect

of electrical shock on 105 
effect on 31 -; implantation in hypothalamus 83,129 -, neurosecretion, effect on 83 -, pituitary-adrenal function, effect

on 322 Ether, pituitary-adrenal function,

effect on 184

Feedback, 'internal',

to hypothalamus 350 Frog larvae, hypothalamic

neurosecretory system in 45

Genetic-neuroendocrine interactions,

(ole in metamorphosis 293 Gold thioglucose, luteotrophic-

lactogenic effects of 93 Growth, somatic, effect of hypothalamic

lesions on 265 Growth hormone-releasing factor,

separation of 34

Historical data; neuroendocrinology 1 Hydrocortisone, v. Cortisol 11-Hydroxycorticoids; in depressive

illness 358 -Hydroxytryptamine in brain and

pineal, endocrine effects on 2515 -Hydroxytryptophan decarboxylase

in developing toad 257 Hyperostosis cranii, v. Endocraniosis Hypophysectomy;

-; subcommissural organ effect on 178 -; subtemporal, technique of,

in monkey 313 Hypothalamic neurons, pituitary

hormones, effect on 350 Hypothalamic-pituitary system; -, gold thioglucose, effect on 93 -; vascular connections 193, 214, 303 Hypothalamus; -, EEG, in female rat 31 -, implantation of corticoids in 129, 228 -, implantation of estrogen in 83,129 -, implantation of reserpine in 228 -, implantation of thyroxine in 15 
Subject Index

-, lesioning of; effect on growth and obesity 265

-, lesioning of; effect on pituitary-thyroid system 158

-, neurosecretion in, effect of estrogen on 83

, neurosecretion in; in frog larvae 45

, perivascular structures in; in man 166

; role in gonadal feedback systems 83

, thyrotrophin-releasing factor in 4

-, thyroxine sensitivity of; in tadpoles 293

Light, subcommissural organ,

effect on 178 Liver enzymes, stress and cortisol

effect on 138 Luteinizing hormone in pituitary,

effect of puberty on 333

Mammary stimulation; in gold-thioglucose treated mice 93

Median eminence, ultrastructure of 214

Metabolic cranio

Metamorphosis;

, neuroendocrine-genetic interactions, role in 293

Morgagni's syndrome, v. Endocraniosis

Neuroendocrine-genetic interactions; role in metamorphosis 293

Neuroendocrinology; historical data 1

Neurosecretion in hypothalamus, effect of estrogen on 83

Neurosecretory granules, ultrastructure of 214

Neurosecretory materials; in subcommissural organ of teleosts 178

Neurosecretory nerve endings, ultrastructure of 214

Neurosecretory nuclei in hypothalamus, perivascular structures of 166

Neurosecretory system of hypothalamus ; in frog larvae 45

Obesity; induction by hypothalamic

lesions 265 Obituary; ERNST A. SCHARRER 65 Ovarian hypertrophy, hypothalamic

steroid implants, effect on 129 
Pentobarbital, pituitary-adrenocortica

system, effect on 184 Pineal gland, 5-hydroxytryptamine

251 Pititary;-; ectopic graft, sensility to

thyroid hormone 276 -, luteinizing hormone in 333 -, pars intermedia, blood supply of 293 -, portal circulation of 193, 214, 303 Pituitary-adrenal system; -, anesthetic agents, effect on 184 -, estrogen, effect on 322 -, hypothalamic corticoid

and reserpine

mplants, effect on 228 -, hypothalamic thyroxine implan

effect on 15 Pituitary-gonadal feedback system, role

of hypothalamus in 83 Pituitary hormones, hypothalamic

neurons, effect on 350 Pituitary-thyroid system; - -, hypothalamic lesions, effect on 158 -, regulation of 276 Phenoxybenzamine, neurotropic

pituitary, effect on 333

Reproductive functions, gold-

hịoglucose, effect on 93 Reserpine; implantation in

hypothalamus 228

SCHARRER, ERNST A., obituary 65 Sexual behavior, hormones, effect on 144 Stress; induced by electrical shock in

man 105 -, liver enzymes, effect on 138 -; 'social' in wild rat; effect on

thyroid 113 Subcommissural organ, secretory

activity of; in teleosts 178

Testosterone, sexual behavior, effect

on 144 Thyroid feedback 276, 293 Thyroid function; -, neonatally administered thyroxine,

effect on 121 -, regulation of 276 -, 'social stress', effect on 113

Subject Index 
Thyrotrophin-releasing factor;

concentration in hypothalamus 4 Thyrotrophin secretion; -; from ectopic pituitary grafts 276 -, regulation of 4 Thyroxine; -, ectopic pituitary grafts, sensitivity to 276 -; implantation in hypothalamus 15 -, neonatal administration, effect of 121 
sensitivity of hypothalamus to; in

tadpole 293 -, thyrotrophin secretion, effect on 4 Transaminase; in liver 138

Vaginal stimulation, hypothalamic

potentials, effect on 31 Vascular system; in hypothalamic-pitu-

potentials, effect on 31 Vascular system; in hypothalamic-pitu-
itary region 193, 214, 303 Vasopressin, blood flow, effect on 23 